\title{
ACUTE PERITONITIS SECONDARY TO SYSTEMIC LUPUS ERYTHEMATOSUS (SLE)
}

Victoria Silveira de Carvalho1,*, Ana Laura Fischer Kunzler', Maurício Simoni Candaten"1, Andrea Worm Furtado', Afonso Guilherme Schmidt, Larissa Vargas Cruz', Charles Lubianca Kohem', Natália Sarzi Sartori'

1. Hospital de Clínicas de Porto Alegre, Porto Alegre (RS), Brazil.

*Corresponding author: victoriacarvalhomd@gmail.com

\section{BACKGROUND}

Among the symptoms resulting from the involvement of the gastrointestinal tract in systemic lupus erythematosus (SLE), ascites occurs in about 8 to $12 \%$ of adult patients and in $55 \%$ of those who complain of abdominal pain, which may be due to causes such as infarction, visceral perforation, pancreatitis, mesenteric vasculitis, hemorrhage, bacterial peritonitis and peritonitis secondary to SLE, which is rare. However, this condition can develop quickly in a flare of lupus. The diagnostic investigation must be guided by abdominal imaging and analysis of the ascitic fluid, which will show an exudative pattern.

\section{CASE REPORT}

Female patient, 30 years old, diagnosed with mild SLE and no history of visceral involvement, using hydroxychloroquine and methotrexate for refractory arthritis, was admitted due to 5 days of abdominal distension, epigastric pain, nausea, vomiting and fever. Abdominal imaging showed a moderate volume of ascites and no hepatic or vascular abnormalities. She had active arthritis and low complements levels, positive Anti-dsDNA, anemia, leukopenia, lymphopenia and thrombocytopenia. There was no serositis elsewhere neither signs of kidney, heart or neurological activity. Paracentesis showed low serum-to-ascites albumin gradient (SAAG) and a high protein level, suggestive of peritoneal disease, with negative cultures and no demonstration of Mycobacterium tuberculosis in peritoneal fluid. Due to suspicion of SLE peritonitis, methylprednisolone $0.5 \mathrm{mg} / \mathrm{kg} / \mathrm{day}$ was started, later increased to $1 \mathrm{mg} / \mathrm{kg} /$ day with no improvement of ascites. Patient underwent a diagnostic laparoscopy with no macroscopic abnormalities in the abdominal cavity, but peritoneal biopsy showed chronic suppurative inflammation with fibrinous exudate and negative cultures. The corticosteroid dose was increased to $2.0 \mathrm{mg} / \mathrm{kg} /$ day, at which point the patient began to show progressive improvement in ascites and hematological activity. She received rituximab and was discharged with clinical and laboratory response, with a gradual reduction in corticosteroid therapy and no return of symptoms.

\section{CONCLUSION}

The diagnostic evaluation of a patient with suspected SLE peritonitis should be careful, since it is extremely important to adequately exclude other causes of an exudative ascites (such as tuberculous or fungal peritonitis and peritoneal carcinomatosis) before initiating immunosuppressive treatment. Lupus peritonitis tends to have a good response to corticosteroid therapy, but the use of other immunosuppressants may sometimes be indicated. In the patient described, rituximab was chosen due to her concomitant hematological and articular activity, with a very good response to therapy. 